\title{
Influence of Self-steepening and Higher Dispersion Effects on the Propagation Characteristics of Solitons in Optical Fibers
}

\author{
Do Thanh Thuy ${ }^{1}$, Nguyen Thanh Vinh ${ }^{1}$, Bui Dinh Thuan ${ }^{1 *}$, Cao Long $\operatorname{Van}^{2}$ \\ ${ }^{1}$ Faculty of Physics and Technology \\ Vinh University, Vietnam \\ ${ }^{2}$ Institute of Physics, University of Zielona Góra \\ A. Szafrana 4a, 65-516 Zielona Góra, Poland \\ *E-mail: thuanbd@vinhuni.edu.vn
}

Received: 08 December 2016; revised: 19 December 2016; accepted: 20 December 2016; published online: 31 December 2016

\begin{abstract}
In this paper we consider the influence of higher-order nonlinear effects like third-order dispersion, self-steepening effect on the propagation characteristics of solitons. By solving the higher-order nonlinear Schrödinger equation we show that the self-steepening effect can lead to the breakup of higher-order solitons through the phenomenon of soliton fission. This effect plays an essential role in several nonlinear phenomena, in particular in the so-called supercontinuum generation in optical fibers. Moreover, we can use third order dispersion to compress pulses as well as changing the frequency.
\end{abstract}

Key words: self-steepening effect, third order dispersion, soliton, supercontinuum generation

\section{INTRODUCTION}

Soliton in optical fibers is formed by the balance between self-phase modulation and dispersion caused by group velocity. Optical solitons are not only predicted theoretically [1-4] but they have also been observed experimentally [5,6]. Some possible applications of solitons such as optical pulse compression, all optical switching, logic devices, etc., have been proposed. The low-loss transmission of soliton in optical fibers is described by the non-linear Schrödinger equation. However, when pulse's width $T_{0}<1 \mathrm{ps,} \mathrm{in} \mathrm{modeling} \mathrm{the}$ propagation of ultrashort pulses, the higher order effects in nonlinear media should be taken into account, and therefore the governing equation should still include third-order dispersion (TOD) and self-steepening [7, 8]. The effect of TOD is significant for fs pulses when the GVD is close to zero [1,6]. The Raman scattering can lead to the self-steepening effect, which can lead further to the breakup of higher-order solitons called soliton fission. This effect has an important contribution in the supercontinuum generation (SG) which is recently a flagship application of photonic crystal fibers [10]. Injected monochromatic pulse may be dramatically broadened (spectrally), which creates a coherent beam generation of high brightness comparable to that of monochromatic lasers. In optics, a supercontinuum is formed when a collection of nonlinear processes act together upon a pump beam in order to cause severe spectral broadening of the original pump beam, for example using a microstructured optical fiber. One of these processes is just the soliton fission.

Such beams have wide applications, for example in trace gas sensing in classical absorption spectroscopy, cavityenhanced absorption spectroscopy, cavity ring-down spectroscopy, and in a diverse range of fields, including optical coherence tomography, frequency metrology, fluorescence lifetime imaging, optical communications, and many others. Therefore, consideration of soliton fission is very important for better understanding of the SG mechanism.

In this work we use the generalized nonlinear Schrödinger equation to study propagation of ultrashort optical pulses in the presence of Raman scattering and third - order dispersion effects. A systematic derivation of this equation has been presented in [11]. The numerical calculations will show that the 
envelope function becomes asymmetric and splits to several subpulses during the propagation, so we have soliton fission.

\section{THEORY}

To study the influence of higher-order dispersion, self phase modulation and self-steepening effects on the propagation dynamics, we use a generalized scalar nonlinear Schrodinger equation (GNLSE) to model the pulse propagation inside the fiber [11]

$$
\begin{aligned}
& i \frac{\partial A(z, t)}{\partial z}+i \beta_{1} \frac{\partial A(z, t)}{\partial t}-\frac{\beta_{2}}{2} \frac{\partial^{2} A(z, t)}{\partial t^{2}} \\
& \quad-\frac{i \beta_{3}}{6} \frac{\partial^{3} A(z, t)}{\partial t^{3}}+\gamma|A(z, t)|^{2} A(z, t) \\
& \quad+\left(\frac{\gamma i}{\omega_{0}} \frac{\partial|A(z, t)|^{2}}{\partial t}-\gamma T_{R} \frac{\partial|A(z, t)|^{2}}{\partial t}\right) A(z, t)=0
\end{aligned}
$$

where $A=A(z, t)$ is a complex envelop function of the optical field. This function varies slowly with time and $\mathrm{z}$ position along the optical fiber; $\beta_{1}, \beta_{2}$ and $\beta_{3}$ are first, second and third-order dispersion factors, respectively; $\gamma$ is the nonlinear factor of the optical fiber; $\gamma T_{R}$ term describes the Raman scattering effect. The term having the factor $1 / \omega_{0}$ is related to the self-steepening effect.

We can normalize equation (1) in the following way:

$$
\begin{gathered}
U(\xi, \tau)=\frac{1}{\sqrt{P_{0}}} A(z, t), \quad L_{D}=\frac{\tau_{0}^{2}}{\left|\beta_{2}\right|} \\
L_{N}=\frac{1}{\gamma P_{0}}, \quad N^{2}=\frac{\tau_{0}^{2} \gamma P_{0}}{\left|\beta_{2}\right|}, s=\frac{1}{\omega_{0} \tau_{0}} \\
\tau=\frac{t-\beta_{1} z}{\tau_{0}}, \quad \xi=\frac{z}{L_{D}}, \quad \delta_{3}=\frac{\beta_{3}}{6\left|\beta_{2}\right| \tau_{0}}
\end{gathered}
$$

where $\mathrm{L}_{D}$ is the dispersive length, $L_{N}$ is the nonlinear length, $\tau_{0}$ is the pulse width, $P_{0}$ is the peak power of the input pulse, and $\mathrm{N}$ characterizes the soliton order that provides the measure of the strength of the nonlinear response compared to the fiber dispersion. We can rewrite the equation (1) in the normalized form:

$$
\begin{gathered}
\frac{\partial U}{\partial \xi}=\frac{i}{2} \frac{\partial^{2} U}{\partial \tau^{2}}+\delta_{3} \frac{\partial^{3} U}{\partial \tau^{3}} \\
+i N^{2}\left(|U|^{2} U+i s \frac{\partial}{\partial \tau}\left(|U|^{2} U\right)-\tau_{R} U \frac{\partial|U|^{2}}{\partial \tau}\right)
\end{gathered}
$$

We choose to rewrite the equation (1) in the form (3) because it is more convenient for the numerical calculations. In the general case it is very difficult to find analytic solutions of Eq.(3) and no such solution was known until now. For the numerical solution of Eq. (3) we consider the following expression:

$$
\frac{\partial U(\xi, \tau)}{\partial \xi}=(\widehat{L}+\widehat{N}(U(\xi, \tau))) U(\xi, \tau)
$$

Here $\widehat{L}=\frac{i}{2} \frac{\partial^{2}}{\partial \tau^{2}}+\delta_{3} \frac{\partial^{3}}{\partial \tau^{3}}$ is a linear operator containing time derivative, $\widehat{N}(U)=i N^{2}\left(|U|^{2}+i s \frac{\partial}{\partial \tau}\left(|U|^{2}\right)\right.$ $\left.-\tau_{R} U \frac{\partial|U|^{2}}{\partial \tau}\right)$ is a non-linear operator and is a function of $U(\xi, \tau)$. Equation (4) was solved by using the fourth-order Runge-Kutta method in the interaction picture using the following algorithm $[9,11]$ :

$$
\begin{aligned}
& U_{1}(\xi, \tau)=\operatorname{IFT}\left[\exp \left(\frac{\Delta \xi}{2} \hat{L}(\omega)\right) \operatorname{FT}[U(\xi, \tau)]\right] \\
& K_{1}=\operatorname{IFT}\left[\exp \left(\frac{\Delta \xi}{2} \hat{L}(\omega)\right) \operatorname{FT}\left[\Delta \xi \hat{N} U_{1}(\xi, \tau)\right]\right] ; \\
& K_{2}=\Delta \xi \hat{N}\left(U_{1}(\xi, \tau)+K_{1} / 2\right)\left(U_{1}(\xi, \tau)+K_{1} / 2\right) ; \\
& K_{3}=\Delta \xi \hat{N}\left(U_{1}(\xi, \tau)+K_{2} / 2\right)\left(U_{1}(\xi, \tau)+K_{2} / 2\right) ; \\
& K_{4}=\Delta \xi \hat{N} \\
& \quad \times\left(\operatorname{IFT}\left\{\exp \left(\frac{\Delta \xi}{2} \hat{L}(\omega)\right) \operatorname{FT}\left\{U_{1}(\xi, \tau)+K_{3}\right\}\right\}\right) \\
& \quad \times \operatorname{IFT}\left\{\exp \left(\frac{\Delta \xi}{2} \hat{L}(\omega)\right) \operatorname{FT}\left\{U_{1}(\xi, \tau)+K_{3}\right\}\right\} .
\end{aligned}
$$

We obtain the value of the envelope function in the location $\xi+\Delta \xi$

$$
\begin{gathered}
U(\xi+\Delta \xi, \tau)=\operatorname{IFT}\left\{\exp \left(\frac{\Delta \xi}{2} \hat{L}(\omega)\right) \times\right. \\
\left.\times F T\left[U_{1}(\xi, \tau)+\frac{K_{1}}{6}+\frac{K_{2}}{3}+\frac{K_{3}}{3}\right]+\frac{K_{4}}{6}\right\} .
\end{gathered}
$$

In relations (5) FT and IFT denote the Fourier and inverse Fourier's transforms, respectively. Errors in applying (5) are of orders $(\Delta \xi)^{5}$.

\section{NUMERICAL RESULTS AND DISCUSSION}

In the next numerical simulation we will consider that the shape of initial pulse is defined as:

$$
U(0, \tau)=N \operatorname{sech}(\tau) .
$$

Here $N$ is the order of soliton, for $N=1,2,3$, respectively, the solitons are called fundamental soliton, second-order soliton, and third-order soliton.

In the first case we study numerically by means of the fourth-order Runge-Kutta in the interaction picture method the impact of the self-steepening and self phase modulation effects on the propagation characteristic of ultrashort pulses. In this case, $\beta_{2}=0, \beta_{3}=0$, so we need to define a normalized distance as $\xi=\frac{z}{L_{N}}$, the pulse propagation equation reduces to the form

$$
\frac{\partial U}{\partial \xi}=i N^{2}\left(|U|^{2} U+i s \frac{\partial}{\partial \tau}\left(|U|^{2} U\right)\right) .
$$




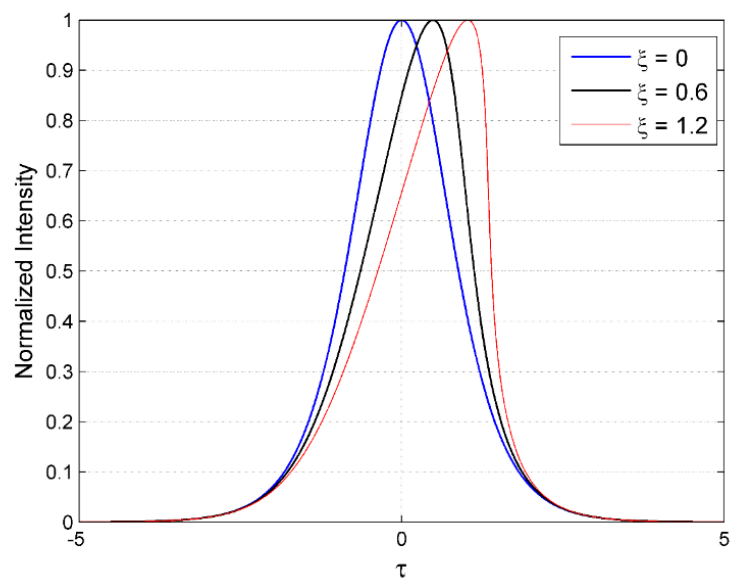

Fig. 1. Influence of self-steepening and self phase modulation effect on the propagation characteristic of ultrashort pulses

Fig. 1 shows the pulse shapes at $\xi=0, \xi=10$ and $\xi=20$ for $s=0.3$ and $N=1$. From this figure we see that when the pulse propagates inside the fiber, it becomes asymmetric, with its peak shifting toward the trailing edge. Therefore, the self-steepening can create an optical shock on the trailing edge in the absence of GVD effects. This is due to the intensity dependence of the group velocity such that the peak moves at a lower speed than the wings.

Next, we investigated the changes of pulse under the influence of second order dispersion to the self-steepening effect. Fig. 2 shows propagation of the ultrashort pulse with the initial hyperbolic secant shape for $s=0.3$, and $N=1$ over the distance $\xi=50$. From this figure we see that the GVD dissipates the shock and smoothens the trailing edge considerably. For fundamental-order soliton the steepening of the trailing edge is not very prominent and the intensity of the peak pulse is constant during transmission. However, the self-steepening still expressed through the change in the pulse center.

Fig. 3 shows the results for the case when the nonlinear parameter $\mathrm{s}=0.3$ and the ultrashort input pulse has different powers. The numerical calculations show that the envelope function becomes asymmetric and splits to several subpulses during the propagation. Thus, the influence of the self-steepening on the propagation of higher order-solitons in optical fibers is the breakup of such solitons into their constituents, a phenomenon known as soliton fission.

Now we consider the case when the pulse wavelength lies in the vicinity of the zero-dispersion wavelength, the $\beta_{3}$ term provides the dominant contribution to the dispersive effects. In this case we can not use the above mentioned normalization. To normalize the equation (1), we must choose scaling parameters as follows

$$
\xi=\frac{z}{L_{D}^{\prime}}, L_{D}^{\prime}=\frac{\tau_{0}^{3}}{\left|\beta_{3}\right|}, N=\frac{\gamma P_{0} \tau_{0}^{3}}{\left|\beta_{3}\right|}
$$

and equation (6) is rewritten

$$
\frac{\partial U}{\partial \xi}=\frac{1}{6} \frac{\partial^{3} U}{\partial \tau^{3}}+i N|U|^{2} U
$$

Fig. 4 corresponds to the case $\beta_{2}=0$ (at the zero dispersion wavelength). The pulse amplitude of the vibration tail which is formed at the back side of pulse decreases along the optical fiber. The maximum of amplitude moves to the positive direction of the axis. Numerical calculation shows that the deformation of pulse caused by third-order dispersion at zero dispersion wavelength can limit the efficiency of optical fibers information system. However, we can use this phenomenon to compress pulses as well as change the frequency.
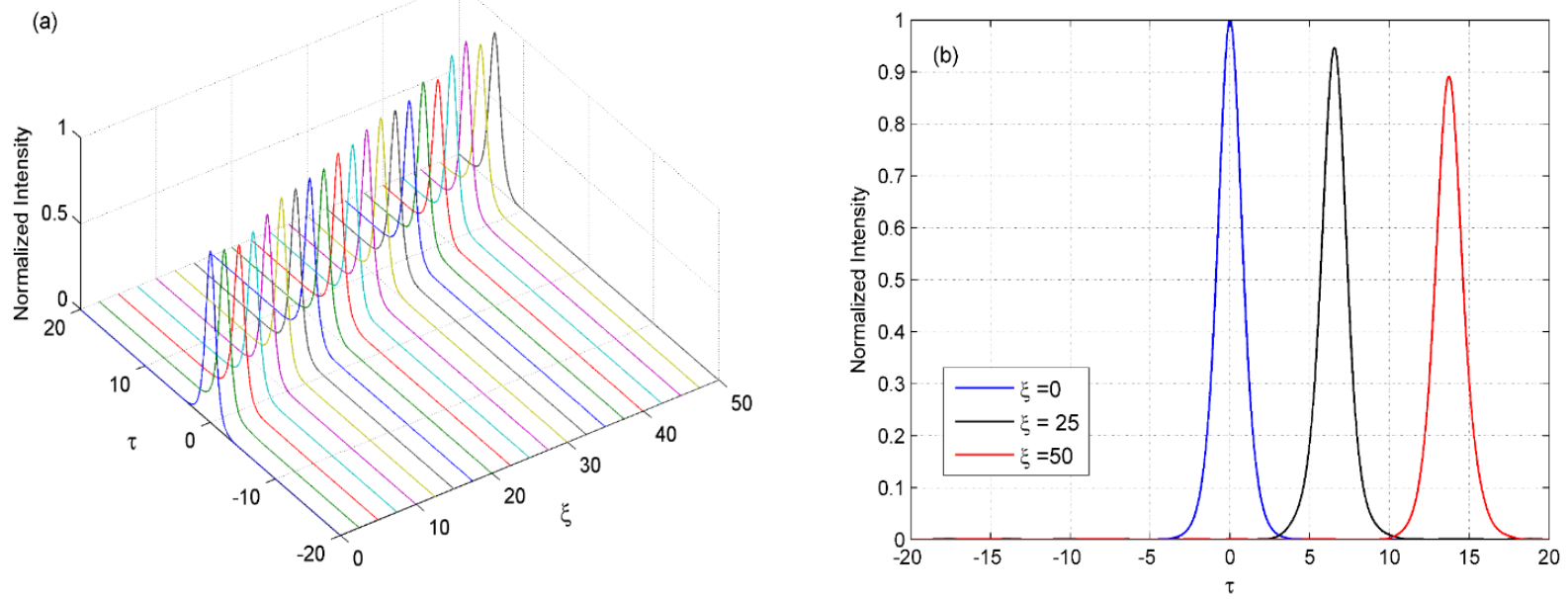

Fig. 2. Propagation of the hyperbolic secant input pulse with the power parameter $N=1$ 

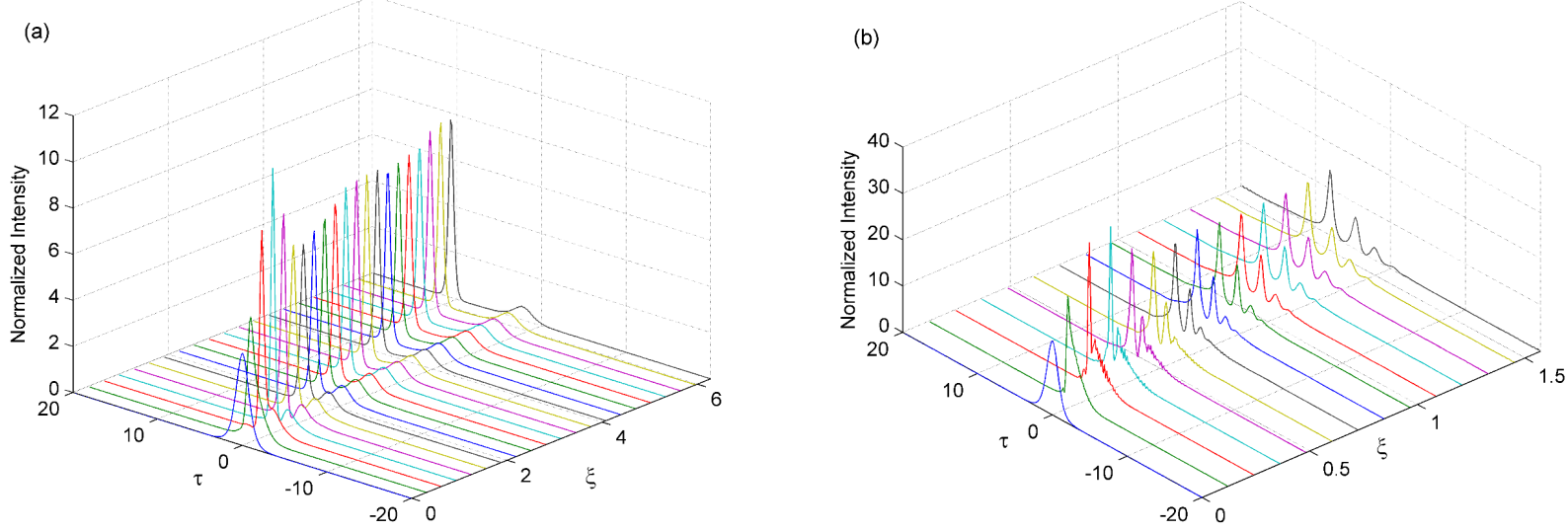

Fig. 3. Propagation of the hyperbolic secant input pulse with the power parameter $N=2$ (a) and $N=3$ (b)

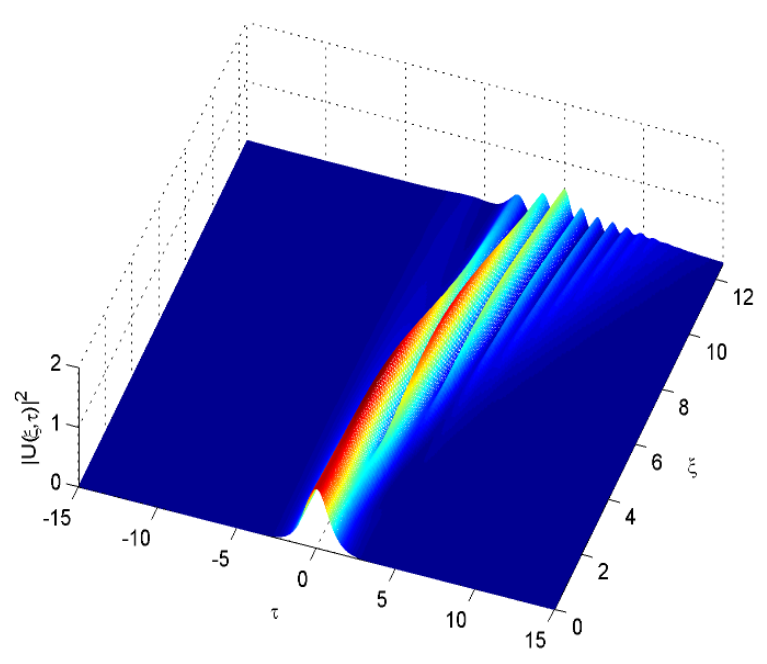

Fig. 4. Propagation of the hyperbolic secant pulse with $\beta_{2}=0$ over the distance $\xi=12$

\section{CONCLUSION}

In this paper we investigate the influence of selfsteepening and higher dispersion effects on the propagation characteristics of solitons in fiber. We show that third order dispersion can lead to a pulse-breakup above a certain pulse power. The influence of the self-steepening on the propagation of higher order-solitons in optical fibers is also investigated, and it is found that self-steepening can lead to the breakup of higher-order solitons into $\mathrm{N}$ fundamental solitons. These generated solitons have higher peak power and shorter temporal duration. They propagate with a higher group velocity and red-shifted [10]. Simultaneously, non-solitonic radiation emitted in the blue wavelength domain of spectrum creates a channel to lose energy. As a result, the phase-matching conditions for the red-shifting solitons are fulfilled, which leads to stimulating several other nonlinear effects as fourwave mixing and cross-phase modulation. All these nonlinear effects are able to fill the remaining gaps in the spectrum, so a flat and broad spectrum can be obtained as white light called supercontinuum. Thus our results concerning soliton fission will be used in a qualitative explanation of supercontinuum generation in our future paper [12].

\section{Acknowledgements}

This research was funded by Vietnam National Foundation for Science and Technology Development (NAFOSTED) under grant number 103.03-2014.62.

\section{References}

[1] G.P. Agrawal. Nonlinear Fiber Optics, Academic, San Diego, 2003.

[2] P.N. Butcher and D. Cotter, The Elements of Nonlinear Optics, Cambridge University Press, 1991.

[3] Y.S. Kivshar, G. P. Agrawal, Optical Solitons, 2003.

[4] J.H.B. Nijhof, H.A. Ferwerda, and B.J. Hoenders, Derivation of the equation for an ultrashort pulse in a fibre, Pure Appl. Opt. 4, 199-218 (1994).

[5] A. Hasegawa and Y. Kodama, Solitons in optical communication, Oxford University Press, New York, 1995.

[6] G.P. Agrawal and M.J. Potasek, Nonlinear pulse distortion in single mode optical fibers at the zero-dispersion wavelength, Phys Rev 33(3), 1765-1776 (1986).

[7] M. Facăo, M. I. Carvalho, Soliton self-frequency shift: Selfsimilar solutions and their stability, Physical Review E 81, 046604 (2010).

[8] H. P. Tian, Z. H. Li, Z. Y. Xu, J. P. Tian, and G. S. Zhou, Stablesoliton in the fiber-optic system with self-frequency shift, J. Opt.Soc. Am. B 20, 59-64 (2003).

[9] Zhongxi Zhang, Liang Chen, and Xiaoyi Bao, A fourth-order Runge-Kutta in the interaction picture method for numerically solving the coupled nonlinear Schrödinger equation, Optics Express 8, 8261-8276 (2010). 
[10] J.M. Dudley, J.R.Taylor, Supercontinuum Generation in optical fibers, Cambridge, 2010.

[11] Van Cao Long, Rev. Adv. Mater. Sci. 23 8-24 (2010).
[12] Do Thanh Thuy, Bui Dinh Thuan, Dinh Xuan Khoa, Nguyen Thanh Vinh, Cao Long Van, to be published.

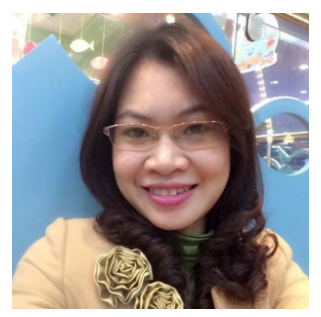

Do Thanh Thuy was born in Nghe An province, Vietnam. She graduated in Physics from Vinh University in 2003. She completed her MSc study in Vinh University in 2005. Presently, she is completing her PhD dissertation at the Faculty of Physics and Technology, Vinh University, under the supervision of professor Dinh Xuan Khoa. She is interested in quantum and nonlinear optics.

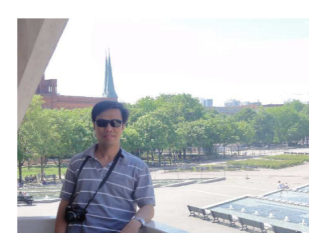

Nguyen Thanh Vinh was born in Nghe An province, Vietnam, He graduated in Physics in 1995 and finished his MSc study in 2005 at the Vinh University. In 2014 he defended his PhD dissertation entitled "Cellular Automata simulations for the system of two-level atoms placed in two-dimensional cavity" at the Faculty of Physics and Astronomy, University of Zielona Góra under supervision of professor Cao Long Van. He is interested in quantum and nonlinear optics, recently he is continuing his work at the Vinh University as a lecturer.

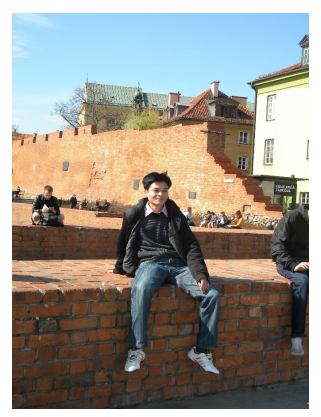

Bui Dinh Thuan was born in Nghe An province, Vietnam, He graduated in Physics in 2002 and completed his MSc study at the Vinh University in 2004. In 2013 he defended his PhD dissertation entitled "Structured continuum effects on optical processes" at the Faculty of Physics and Astronomy, University of Zielona Góra, under supervision of professor Wiesław Leoński. Presently, he is continuing his work at the Vinh University as a lecturer. His research interests concern quantum and nonlinear optics.

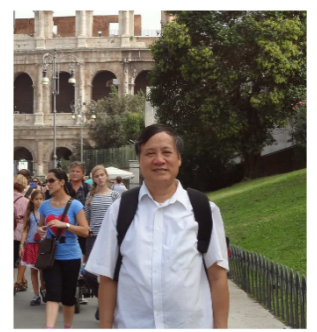

Cao Long Van was born in 1952 in Hanoi, Vietnam. He graduated from Warsaw University with an MSc degree in Theoretical Physics in 1976. In 1979 he defended his PhD dissertation at this University. In 1987 he habilitated in the Center of Theoretical Physics, Polish Academy of Sciences in Warsaw with a thesis entitled "Noises in Quantum Optics". He is presently professor of the University of Zielona Góra. His research interests concern several topics in Quantum and Nonlinear Optics. 